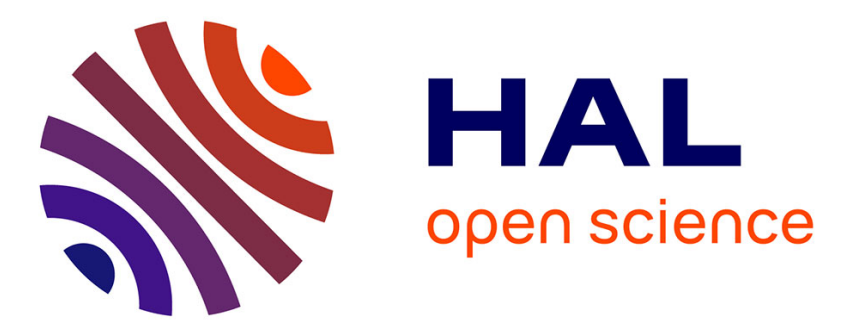

\title{
An Average Flow Model of the Reynolds Roughness Including a Mass-Flow Preserving Cavitation Model
}

Guy Bayada, Sébastien Martin, Carlos Vazquez

\section{To cite this version:}

Guy Bayada, Sébastien Martin, Carlos Vazquez. An Average Flow Model of the Reynolds Roughness Including a Mass-Flow Preserving Cavitation Model. 2004. hal-00008082

\section{HAL Id: hal-00008082 \\ https://hal.science/hal-00008082}

Preprint submitted on 20 Aug 2005

HAL is a multi-disciplinary open access archive for the deposit and dissemination of scientific research documents, whether they are published or not. The documents may come from teaching and research institutions in France or abroad, or from public or private research centers.
L'archive ouverte pluridisciplinaire HAL, est destinée au dépôt et à la diffusion de documents scientifiques de niveau recherche, publiés ou non, émanant des établissements d'enseignement et de recherche français ou étrangers, des laboratoires publics ou privés. 


\title{
AN AVERAGE FLOW MODEL OF THE REYNOLDS ROUGHNESS INCLUDING A MASS-FLOW PRESERVING CAVITATION MODEL
}

\author{
(September 2004)
}

\author{
Guy Bayada \\ INSA Lyon, France \\ LAMCOS CNRS-UMR 5514 / MAPLY CNRS-UMR 5585 \\ Bat. L. de Vinci, 21 av. Jean Capelle, \\ 69621 Villeurbanne cedex, France \\ Email: guy.bayada@insa-lyon.fr \\ Sébastien Martin \\ INSA Lyon, France \\ MAPLY CNRS-UMR 5585 \\ Bat. L. de Vinci, 21 av. Jean Capelle, \\ 69621 Villeurbanne cedex, France \\ Email: sebastien.martin@insa-lyon.fr \\ Carlos Vázquez \\ Universidade da Coruña \\ Facultade de Informática / Dept. de Matemáticas \\ Email: carlosv@udc.es
}

\begin{abstract}
An average Reynolds equation for predicting the effects of deterministic periodic roughness, taking JFO mass flow preserving cavitation model into account, is introduced based upon double scale analysis approach. This average Reynolds equation can be used both for a microscopic interasperity cavitation and a macroscopic one. The validity of such a model is verified by numerical experiments both for one dimensional and two dimensional roughness patterns.
\end{abstract}




\section{NOMENCLATURE}

$A_{\varepsilon}, B_{\varepsilon}, A_{i}, B_{i}$

$a_{i j}^{\star}, a_{i}^{\star}, a_{i}^{0}=$

$A_{i j}^{\star}, B_{i}^{\star}, B_{i}^{0}$

$h_{1}, h_{2}$

$h, h_{\varepsilon}$

$h_{s}$

$h_{r}$

$p$

$p_{0}, p_{1} \ldots$

$Q$

$U$

$x=\left(x_{1}, x_{2}\right)$

$y=\left(y_{1}, y_{2}\right)$

$X=\left(X_{1}, X_{2}\right)$

$X^{\prime}=\left(X_{1}^{\prime}, X_{2}^{\prime}\right)$

$Y=] 0,1[\times] 0,1[$

$\gamma$

$\partial / \partial n$

$\mu$

$\varepsilon$

$\theta$

$\theta_{0}$

$\Theta, \Theta_{1}, \Theta_{2}$

$w_{i}, \chi_{i}^{0}$

$-Y$

$[\cdot]_{Y_{1}}$

$[\cdot]_{Y_{2}}$
$=$ partial differential operators

$=$ auxiliary homogenized coefficients

$=$ homogenized coefficients

$=$ description of the gap

$=$ actual gap

$=$ smooth part of the gap

$=$ amplitude of the roughness

$=$ pressure

$=$ approximations of the pressure

$=$ input flow value

$=$ velocity

$=$ dimensionless space coordinates

$=$ microscale coordinates

$=$ oblique coordinates

$=$ real coordinates

$=$ rescaled microcell

$=$ obliqueness angle

$=$ normal derivative

$=$ viscosity

$=$ roughness spacing

$=$ saturation

$=$ microscopic homogenized saturation

$=$ macrohomogenized saturations

$=$ auxiliary functions defined on $Y$

$=$ average operator with respect to $y$

$=$ average operator with respect to $y_{1}$

$=$ average operator with respect to $y_{2}$

\section{Introduction}

The effects of the surface roughness on the behavior of a thin film flow has long been the subject of intensive studies. Various ways have been introduced to study Reynolds roughness by seeking an average equation with smooth coefficients. Some of the most popular results are the Christensen formula [1] for longitudinal and transverse roughness and the Patir and Cheng flow factor model [2] for a more general surface roughness pattern. Two wide classes of results can be outlined. In the first one, which is deterministic, a periodic description of the surfaces is often assumed to be known and linked to a specific process of the surface [3]. It is possible to distinguish macrovariables and microvariables and to use a mathematical homogenization approach to rigorously obtain an average Reynolds equation by making the period of the roughness tend to zero [4]. The coefficients of this average Reynolds equation implicitly contain the description of the microroughness elementary cell. The second class of results deals with a statistical description of the surface roughness. Following the Patir and Cheng approach, numerous authors proposed an average Reynolds equation in which the coefficients included the knowledge of the surface statistics by way of flow factors which can be evaluated by numerical experiments. Rigorously speaking, this approach is less satisfactory than the first one, assuming a priori the existence of a control volume in which 
the average flow rates can be equivalently expressed in terms of flow factors. The number and quantities (Peklenik number, combined root mean square roughness...) involved in the characterization of the flow factors can also be discussed. Moreover, as the initial Reynolds equation, the average Reynolds equation can be expressed in terms of $\nabla \cdot(K \nabla p)=F$ in which $K$ is a diagonal matrix. This seems to be contradictory with the result obtained by the first approach in which $K$ is a non diagonal matrix for two dimensional general roughness pattern [5].

Up to now, these averaging processes never take cavitation into account. A common procedure is to use the average equation instead of the classical Reynolds equation with Gumbel and Swift Steiber boundary conditions or to include it in the S.O.R. algorithm proposed by Richardson, thus obtaining the splitting of the lubricated device in two areas. In a first area, the pressure is greater than the cavitation pressure and the average Reynolds equation is valid; in the other area, pressure is equal to the cavitation pressure. It is well known [6-8], however, that none of these models is mass preserving, especially through the cavitation area. Jakobsson, Floberg and Olsson (JFO) $[9,10]$ developed a set of conditions for the cavitation boundary that properly takes the conservation of mass into account in the entire device. Elrod $[11,12]$ proposed a slightly modified formulation and a related specific algorithm. The mathematical related problem evidence a hyperbolic-parabolic feature which renders difficult both theoretical study and numerical experiments [7, 13-15]. It is the goal of this paper to develop in a rigorous way an average JFO Reynolds equation for the deterministic periodic roughness pattern. So far, few papers have been devoted to such a problem. Recently, the interasperity cavitation has been studied by way of a statistical approach $[16,17]$. The Patir and Cheng flow factor method is extended and an average Reynolds equation is proposed. The resulting equation has the same left-hand side that in the Patir and Cheng equation (cavitation has no effect on the corresponding flow factors) while the right-hand side of the equation is modified and new flow factors are introduced. At last Harp and Salant [17] proposed to modify the boundary conditions by a value which is a function of the wavelength of the roughness. Our approach is quite different and explicitly based upon the introduction of fast and slow variables. The initial equation is rewritten in terms of these two variables and asymptotic expansion of the pressure is introduced with respect to a small parameter associated to the roughness wavelength. The goal is to find an equation satisfied by the first terms of the expansion. Some assumptions about the shape of the roughness appear to be necessary to solve the problem, leading to a new average Reynolds cavitation equation. This equation has numerous common features with the initial Reynolds equation: it is also a two unknowns pressure-saturation formulation. Some particular cases - transverse, longitudinal roughness patterns will be studied in details.

\section{Basic equations}

Our studied cavitation model, like the Elrod algorithm and its variants, views the film as a mixture. It does not, however, make the assumption of liquid compressibility in the full film area as in [15] and some other papers. As in $[18,19]$, only the liquid-vapor mixture in the cavitated region is assumed compressible. The flow obeys the following "universal" Reynolds equation (here written in a dimensionless form) through all the gap in which the pressure cavitation is assumed to be zero in the cavitation area

$$
\begin{gathered}
\sum_{i=1}^{2} \frac{\partial}{\partial x_{i}}\left(h^{3} \frac{\partial p}{\partial x_{i}}\right)=\frac{\partial \theta h}{\partial x_{1}}, \\
p \geq 0 \\
0 \leq \theta \leq 1
\end{gathered}
$$




$$
p(1-\theta)=0 .
$$

In this steady state isoviscous version of the equation, $p$ is the pressure, $\theta$ is the relative mixture density, $h$ the film thickness, $x_{1}$ is the direction of the effective relative velocity of the shaft, while $x_{2}$ is the transverse direction.

This system of equations can be understood as follows (see [7,9-12,14,18] for various comments and meaning of the $\theta$ variable):

- the well-known Reynolds equation holds in the full film region, that is $p>0$ and $\theta=1$,

- a mass flow conserving equation $\partial \theta h / \partial x_{1}=0$ holds in the cavitated region with $p=0$ and $0<\theta<1$.

- a boundary condition which is also mass flow preserving at the (unknown) interface between the two regions:

$$
-h^{3} \frac{\partial p}{\partial n}+h \cos \left(n, x_{1}\right)=\theta h \cos \left(n, x_{1}\right)
$$

The reason to retain this specific cavitation equation is that it has been the subject of numerous mathematical studies [7] giving a strong and rigorous basis to the following manipulations [20]. To be noticed, however, that our approach can be applied without difficulty to other cavitation models as the one in [15]. Last, it has to be mentioned that this equation takes both macrocavitation (associated to the occurrence of a diverging part of a bearing for example) and interasperity cavitation into account.

The boundary conditions depend on the considered device. However, the following ones are often used, corresponding for example to a journal bearing with an axial supply groove. The pressure is imposed at two circumferential locations and one axial location. The last boundary condition is an input flow condition at the axial location corresponding to the supply groove:

$$
\theta(x) h(x)-h^{3}(x) \frac{\partial p}{\partial x_{1}}(x)=Q
$$

For small values of $Q$, starvation may occur in the vicinity of the supply groove.

\section{Asymptotic expansion}

Let us suppose that the roughness is periodically reproduced in the two $x_{1}$ and $x_{2}$ directions from an elementary cell $Y$ (or "miniature bearing" in Tonder's terminology). We denote by $\varepsilon$ the ratio of the homothetic transformation passing from the elementary cell $Y=Y_{1} \times Y_{2}$ to the real bearing and by $y_{1}=x_{1} / \varepsilon$ and $y_{2}=x_{2} / \varepsilon$ the local variables (see FIG. 1).

Let us now consider shapes that can be written as $h_{\varepsilon}(x)=h(x, x / \varepsilon)$. We suppose furthermore that they are described as

$$
h_{\varepsilon}(x)=h_{1}\left(x, \frac{x_{1}}{\varepsilon}\right) h_{2}\left(x, \frac{x_{2}}{\varepsilon}\right)
$$

which allows us to take into account either transverse or longitudinal roughness, but also more general two dimensional roughness. Introducing now the fast variables $y_{1}$ and $y_{2}$, it appears that the new expression for the gap 


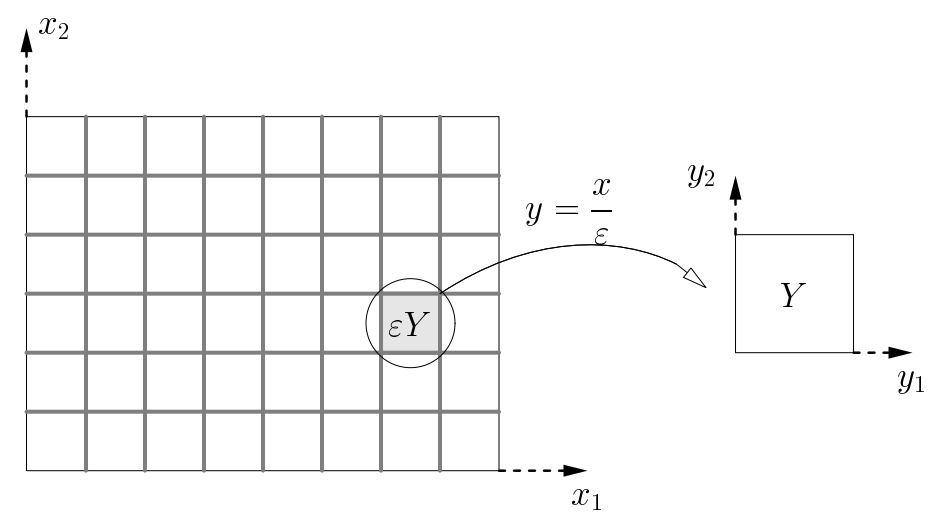

Figure 1. Macroscopic domain and elementary cells

is:

$$
h(x, y)=h_{1}\left(x, y_{1}\right) h_{2}\left(x, y_{2}\right)
$$

The combined computation in terms of $\left(x_{1}, x_{2}\right)$ or $\left(y_{1}, y_{2}\right)$ is an important feature of the method. It is convenient to consider first $x$ and $y$ as independent variables and to replace next $y$ by $x / \varepsilon$ (see [4]).

\subsection{Formulation of average equations}

We denote by $A_{\varepsilon}$ the initial differential Reynolds operator

$$
A_{\varepsilon}[\cdot]=\sum_{j=1}^{2} \frac{\partial}{\partial x_{j}}\left(h^{3}\left(x, \frac{x}{\varepsilon}\right) \frac{\partial[\cdot]}{\partial x_{j}}\right),
$$

and we also define the right-hand side operator

$$
B_{\varepsilon}[\cdot]=\frac{\partial}{\partial x_{1}}\left(h\left(x, \frac{x}{\varepsilon}\right)[\cdot]\right)
$$

The Reynolds equation (1) becomes

$$
A_{\varepsilon}(p)=B_{\varepsilon}(\theta)
$$


The underscript $\varepsilon$ indicates the dependance of the real pressure on the microtexture related to $\varepsilon$. We also define the following operators:

$$
\begin{gathered}
A_{1}[\cdot]=\sum_{j=1}^{2} \frac{\partial}{\partial y_{j}}\left(h^{3}(x, y) \frac{\partial[\cdot]}{\partial y_{j}}\right) \\
A_{2}[\cdot]=\sum_{j=1}^{2} \frac{\partial}{\partial y_{j}}\left(h^{3}(x, y) \frac{\partial[\cdot]}{\partial x_{j}}\right)+\sum_{j=1}^{2} \frac{\partial}{\partial x_{j}}\left(h^{3}(x, y) \frac{\partial[\cdot]}{\partial y_{j}}\right) \\
A_{3}[\cdot]=\sum_{j=1}^{2} \frac{\partial}{\partial x_{j}}\left(h^{3}(x, y) \frac{\partial[\cdot]}{\partial x_{j}}\right)
\end{gathered}
$$

and also

$$
\begin{aligned}
& B_{1}^{i}[\cdot]=\frac{\partial}{\partial y_{i}}(h(x, y)[\cdot]), \quad i=1,2, \\
& B_{2}^{i}[\cdot]=\frac{\partial}{\partial x_{i}}(h(x, y)[\cdot]), \quad i=1,2 .
\end{aligned}
$$

If applied to a function of $(x, x / \varepsilon)$, the operators become

$$
\begin{aligned}
& A_{\varepsilon}=\left(1 / \varepsilon^{2} A_{1}+1 / \varepsilon A_{2}+A_{3}\right), \\
& B_{\varepsilon}=\left(1 / \varepsilon B_{1}^{1}+B_{2}^{1}\right) .
\end{aligned}
$$

We shall look for an asymptotic expansion of the solutions

$$
\begin{aligned}
& p(x)=p_{0}\left(x, \frac{x}{\varepsilon}\right)+\varepsilon p_{1}\left(x, \frac{x}{\varepsilon}\right)+\varepsilon^{2} p_{2}\left(x, \frac{x}{\varepsilon}\right)+\ldots, \\
& \theta(x)=\theta_{0}\left(x, \frac{x}{\varepsilon}\right)
\end{aligned}
$$

each unknown $p_{i}$ and $\theta_{0}$ being a function of $(x, y)$. The problem of the boundary conditions to be satisfied by the $p_{i}$ is somewhat difficult but may be summarized as follows.

(i) The natural boundary conditions on $\left(p_{\varepsilon}, \theta_{\varepsilon}\right)$ are assigned to $p_{0}$ and an equivalent saturation linked to $\theta_{0}$, which will be developped in next subsection.

(ii) The function $p_{i}, i \geq 1$, are $Y$ periodic, i.e. periodic in the two variables $y_{1}, y_{2}$, for each value of $\left(x_{1}, x_{2}\right)$.

To be noticed that unlike of $p$, we do not introduce an asymptotic expansion for $\theta$. This can be explained by observing the evolution of $p$ and $\theta$ as $\varepsilon$ tends to 0 (see FIG. 2 for instance). Clearly, the oscillations of the pressure are decreasing and $p$ tends to a smooth function (namely $p_{0}$ which, actually, does not depend on the fast variable as it will be pointed out further). This is not the case for $\theta$ and an asymptotic smooth limit cannot be considered. 
We shall see later that the functions $p_{i}, i \geq 1$, are defined up to an additive constant. Moreover, from Equations (2)-4), the following properties hold:

$$
\begin{gathered}
p_{0}(x, y) \geq 0 \\
0 \leq \theta_{0}(x, y) \leq 1 \\
p_{0}(x, y)\left(1-\theta_{0}(x, y)\right)=0 .
\end{gathered}
$$

Putting Equations (9) and (10) into Equation (1) and taking account of Equations (7) and (8), one can write by an identification procedure:

$$
\begin{gathered}
A_{1} p_{0}=0 \\
A_{1} p_{1}+A_{2} p_{0}=B_{1}^{1} \theta_{0}, \\
A_{1} p_{2}+A_{2} p_{1}+A_{3} p_{0}=B_{2}^{1} \theta_{0} .
\end{gathered}
$$

Let us remark that these equations are of the following type: For a given $F$, find a function $q$, depending on the variable $y, q$ being $Y$ periodic, such that ( $x$ is a parameter),

$$
A_{1} q=F
$$

A condition to have a solution for Equation (17) is

$$
\int_{Y} F(x, y) d y=0
$$

Moreover, if $q$ is a solution, then $q+c$ with $c$ any constant with respect to $y$ is also a solution. Applying Condition (18) to Equation (14), we deduce that $p_{0}$ does not depend on $y$

$$
p_{0}(x)
$$

Let us suppose now that $p_{0}$ is known, and noticing that, due to boundary conditions, $\left(B_{1}^{1} \theta_{0}-A_{2} p_{0}\right)$ satisfies Equation (18), existence of $p_{1}$ is guaranteed. Now we can represent $p_{1}$ as a function of $p_{0}$ in a more usable form. We define $w_{i}$ and $\chi_{i}^{0}(i=1,2)$ as the $Y$ periodic solutions (up to an additive constant) of the following local problems:

$$
\begin{aligned}
& A_{1} w_{i}=\frac{\partial h^{3}}{\partial y_{i}}, \quad i=1,2 \\
& A_{1} \chi_{i}^{0}=\frac{\partial \theta_{0} h}{\partial y_{i}}, \quad i=1,2 .
\end{aligned}
$$


The solution of Equation (15) reduces to

$$
p_{1}(x, y)=\chi_{1}^{0}(x, y)-\frac{\partial p_{0}}{\partial x_{1}}(x) w_{1}(x, y)-\frac{\partial p_{0}}{\partial x_{2}}(x) w_{2}(x, y)
$$

The same procedure can be used to ensure the existence of $p_{2}$, but in that step, the corresponding condition (18) applied to Equation (16) becomes

$$
\int_{Y}\left(B_{2}^{1} \theta_{0}-A_{2} p_{1}-A_{3} p_{0}\right) d y=0
$$

Then the main idea is to put Equation (22) into Equation (23), so that the only remaining unknowns are $p_{0}$ and $\theta_{0}$.

By analogy with the probabilistic framework, we denote by $\bar{u}^{Y}$ the local average of any $Y$ periodic function $u$ :

$$
\bar{u}^{Y}(x)=\frac{1}{[Y]} \int_{Y} u(x, y) d y
$$

By exchanging the integral and the derivation symbols, and after some calculations, Equation (23) becomes

$$
\sum_{i, j} \frac{\partial}{\partial x_{i}}\left(A_{i j}^{\star} \frac{\partial p_{0}}{\partial x_{j}}\right)=\left(\frac{\partial B_{1}^{0}}{\partial x_{1}}+\frac{\partial B_{2}^{0}}{\partial x_{2}}\right),
$$

where $(i, j=1,2$ and $j \neq i)$

$$
\begin{aligned}
& A_{i i}^{\star}={\overline{h^{3}}}^{Y}-{\overline{h^{3} \frac{\partial w_{i}}{\partial y_{i}}}}^{Y} \\
& A_{i j}^{\star}=-{\overline{h^{3} \frac{\partial w_{j}}{\partial y_{i}}}}^{Y}=-{\overline{h^{3} \frac{\partial w_{i}}{\partial y_{j}}}}^{Y}=A_{j i}^{\star},
\end{aligned}
$$

and also

$$
\begin{aligned}
& B_{1}^{0}={\overline{\theta_{0} h}}^{Y}-\frac{{\overline{h^{3} \frac{\partial \chi_{1}^{0}}{\partial y_{1}}}}^{Y}}{B_{2}^{0}=} \quad-\quad-h^{3} \frac{\partial \chi_{1}^{0}}{\partial y_{2}}
\end{aligned}
$$

Equation (24) deals with any periodic roughness pattern. To be noticed is the fact that the differential operator is no more of the Reynolds type since extra terms $\partial^{2} p_{0} / \partial x_{i} \partial x_{j}$ appear. The right-hand side also contains an additive term in the $x_{2}$ direction. However, the link between $p_{0}$ and $\theta_{0}$ is not so clear. This is a major obstacle which prevents from getting a tractable equation. Nevertheless, Assumption 6 allows us to solve the following difficulties: 
- Computation of $A_{i i}^{\star}, i=1,2$ :

Let us recall Equation (20) with $i=1$ :

$$
\frac{\partial}{\partial y_{1}}\left(h^{3} \frac{\partial w_{1}}{\partial y_{1}}\right)+\frac{\partial}{\partial y_{2}}\left(h^{3} \frac{\partial w_{1}}{\partial y_{2}}\right)=\frac{\partial h^{3}}{\partial y_{1}} .
$$

Since $h^{3} \partial w_{1} / \partial y_{2}$ is $Y$ periodic, averaging this equation over $Y_{2}$ gives

$$
\frac{\partial}{\partial y_{1}}\left(\left[h^{3} \frac{\partial w_{1}}{\partial y_{1}}\right]_{Y_{2}}\right)=\frac{\partial\left[h^{3}\right]_{Y_{2}}}{\partial y_{1}}
$$

where $[\cdot]_{\left[Y_{i}\right]}$ is the averaging operator over $Y_{i}$ (for $i=1,2$ ).

Thus we have, by integrating in the $y_{1}$ variable and using Equations(6):

$$
\left[h^{3}-h^{3} \frac{\partial w_{1}}{\partial y_{1}}\right]_{Y_{2}}=C_{1}
$$

where $C_{1}$ is a constant with respect to $y$. Let us notice that, averaging the earlier equation over $Y_{1}$ simply gives $C_{1}=A_{11}^{\star}$. Thus, it remains to calculate $C_{1}$. Dividing each side of the previous equation by $h_{1}^{3}$ :

$$
\left[h_{2}^{3}\right]_{Y_{2}}-\left[h_{2}^{3} \frac{\partial w_{1}}{\partial y_{1}}\right]_{Y_{2}}=\frac{C_{1}}{h_{1}^{3}}
$$

and, since $w_{1}$ is $Y$ periodic, averaging over $Y_{1}$ gives

$$
A_{11}^{\star}=\frac{{\overline{h_{2}^{3}}}^{\overline{h_{1}^{-3}} Y}}{} .
$$

Following the same procedure, we state:

$$
A_{22}^{\star}=\frac{\overline{h_{1}^{3}} Y}{\overline{h_{2}^{-3}} Y} .
$$

- Computation of $A_{i j}^{\star}, i \neq j$ :

Starting from Equation (20) with $i=1$, since $h^{3}-h^{3} \partial w_{1} / \partial y_{1}$ is $Y$ periodic, averaging this equation over $Y_{1}$ gives

$$
\frac{\partial}{\partial y_{2}}\left(\left[h^{3} \frac{\partial w_{1}}{\partial y_{2}}\right]_{Y_{1}}\right)=0
$$


Thus we have, by integrating in the $y_{2}$ variable

$$
\left[h^{3} \frac{\partial w_{1}}{\partial y_{2}}\right]_{Y_{1}}=C_{2}
$$

where $C_{2}$ is a constant with respect of $y$. Similarly to the computation of $A_{i i}^{\star}$, one has $C_{2}=A_{12}^{\star}=A_{21}^{\star}$. Dividing each side of the equation by $h_{2}^{3}$ :

$$
\frac{C_{2}}{h_{2}^{3}}=\left[h_{1}^{3} \frac{\partial w_{1}}{\partial y_{2}}\right]_{Y_{1}},
$$

and, since $w_{1}$ is $Y$ periodic, averaging over $Y_{2}$ gives $C_{2}{\overline{a_{1}^{-1}}}^{Y}=0$, i.e.

$$
A_{12}^{\star}=A_{21}^{\star}=0
$$

Now, it remains to calculate the right-hand side of the Reynolds equation.

- Computation of $B_{1}^{0}$ :

Let us recall Equation (21) with $i=1$ :

$$
\frac{\partial}{\partial y_{1}}\left(h^{3} \frac{\partial \chi_{1}^{0}}{\partial y_{1}}\right)+\frac{\partial}{\partial y_{2}}\left(h^{3} \frac{\partial \chi_{1}^{0}}{\partial y_{2}}\right)=\frac{\partial \theta_{0} h}{\partial y_{1}}
$$

Since $h^{3} \partial \chi_{1}^{0} / \partial y_{2}$ is $Y$ periodic, averaging this equation over $Y_{2}$ gives

$$
\frac{\partial}{\partial y_{1}}\left(\left[h^{3} \frac{\partial \chi_{1}^{0}}{\partial y_{1}}\right]_{Y_{2}}\right)=\frac{\partial\left[\theta_{0} h\right]_{Y_{2}}}{\partial y_{1}} .
$$

Thus we have, by integrating in the $y_{1}$ variable:

$$
\left[\theta_{0} h-h^{3} \frac{\partial \chi_{0}^{1}}{\partial y_{1}}\right]_{Y_{2}}=C_{3},
$$

where $C_{3}$ is a constant with respect to $y$. Clearly, we have $C_{3}=B_{1}^{0}$. Dividing each side of the equation by $h_{1}^{3}$ :

$$
\left[\frac{\theta_{0} h}{h_{1}^{3}}\right]_{Y_{2}}-\left[h_{2}^{3} \frac{\partial \chi_{1}^{0}}{\partial y_{1}}\right]_{Y_{2}}=\frac{C_{3}}{h_{1}^{3}},
$$


and, since $\chi_{1}^{0}$ is $Y$ periodic, averaging over $Y_{1}$ gives ${\overline{\theta_{0} h / h_{1}^{3}}}^{Y}=C_{3}{\overline{h_{1}^{-3}}}^{Y}$, i.e.

$$
B_{1}^{0}=\frac{\overline{\left(\frac{\theta_{0} h_{2}}{h_{1}^{2}}\right)}}{{\overline{h_{1}^{-3}}}^{Y}}
$$

- Computation of $B_{2}^{0}$ :

Starting from Equation (21) with $i=1$, since the function $h^{3}-h^{3} \partial \chi_{1}^{0} / \partial y_{1}$ is $Y$ periodic, averaging this equation over $Y_{1}$ gives

$$
\frac{\partial}{\partial y_{2}}\left(\left[h^{3} \frac{\partial \chi_{1}^{0}}{\partial y_{2}}\right]_{Y_{1}}\right)=0
$$

Thus we have, by integrating in the $y_{2}$ variable:

$$
-\left[h^{3} \frac{\partial \chi_{1}^{0}}{\partial y_{2}}\right]_{Y_{1}}=C_{4}
$$

where $C_{4}$ is a constant with respect of $y$. We have $C=B_{2}^{0}$. Then dividing each side by $h_{2}^{3}$.

$$
\frac{C_{4}}{h_{2}^{3}}=\left[h_{1}^{3} \frac{\partial \chi_{1}^{0}}{\partial y_{2}}\right]_{Y_{1}}
$$

and, since $\chi_{1}^{0}$ is $Y$ periodic, averaging over $Y_{2}$ gives $C_{4}{\overline{h_{1}^{-3}}}^{Y}=0$, i.e.

$$
B_{2}^{0}=0
$$

Now, it is obvious that Equation (24) can be written in a more simple way by using Equations (25)-(29). Before that, let us write the term $B_{1}^{0}$ in a more usable form. Defining the quantities

$$
\begin{aligned}
B_{1}^{\star} & =\frac{{\overline{h_{1}^{-2}}}^{Y}}{{\overline{h_{1}^{-3}}}^{Y}} \bar{h}_{2}^{Y}, \\
\Theta & =\frac{1}{{\overline{h_{2}}}^{Y} \overline{h_{1}^{-2}} Y} \overline{\left(\frac{\theta_{0} h_{2}}{h_{1}^{2}}\right)} Y,
\end{aligned}
$$

we get $B_{1}^{0}=\Theta B_{1}^{\star}$. Moreover, from Equations (12) and (13), we immediately have:

$$
\begin{gathered}
0 \leq \Theta(x) \leq 1, \\
p_{0}(x)(1-\Theta(x))=0,
\end{gathered}
$$


so that the homogenized equations appear to be

$$
\begin{gathered}
\sum_{i=1}^{2} \frac{\partial}{\partial x_{i}}\left(A_{i i}^{\star} \frac{\partial p_{0}}{\partial x_{i}}\right)=\frac{\partial \Theta B_{1}^{\star}}{\partial x_{1}}, \\
p_{0} \geq 0 \\
0 \leq \Theta \leq 1, \\
p_{0}(1-\Theta)=0,
\end{gathered}
$$

where $A_{11}^{\star}, A_{22}^{\star}$ and $B_{1}^{\star}$ are, respectively, given by Equations (25), (26) and (30). Moreover, the link between a new (smooth) "macroscopic" saturation $\Theta$ and the (oscillating) "microscopic" saturation $\theta_{0}$ is given by Equation (31). As an important feature, $\Theta$ is not the average of the microscopic saturation $\theta_{0}$.

\subsection{Average boundary condition}

When the pressure is imposed, the corresponding average boundary condition is assigned to $p_{0}$. When an input flow is given on a supply line, the average flow condition is obtained following the asymptotic expansion method. Taking account of roughness patterns, Equation (5) becomes:

$$
\theta(x) h\left(x, \frac{x}{\varepsilon}\right)-h^{3}\left(x, \frac{x}{\varepsilon}\right) \frac{\partial p}{\partial x_{1}}(x)=Q
$$

Putting Equations (9) and (10) into Equation (38), one can write by an identification procedure:

$$
\theta_{0}(x, y) h(x, y)-h^{3}(x, y)\left(\frac{\partial p_{0}}{\partial x_{1}}(x)+\frac{\partial p_{1}}{\partial y_{1}}(x, y)\right)=Q
$$

Putting Equation (22) into it gives

$$
\left(\theta_{0} h-h^{3} \frac{\partial \chi_{1}^{0}}{\partial y_{1}}\right)-\left(h^{3}-h^{3} \frac{\partial w_{1}}{\partial y_{1}}\right) \frac{\partial p_{0}}{\partial x_{1}}+\left(h^{3} \frac{\partial w_{2}}{\partial y_{1}}\right) \frac{\partial p_{0}}{\partial x_{2}}=Q
$$

Averaging over $Y$ gives the boundary condition relating $p_{0}$ and $\Theta$ at the supply groove:

$$
B_{1}^{0}-A_{11}^{\star} \frac{\partial p_{0}}{\partial x_{1}}-A_{12}^{\star} \frac{\partial p_{0}}{\partial x_{2}}=Q
$$

and since $A_{12}^{\star}=0$ and $B_{1}^{0}=\Theta B_{1}^{\star}$, one gets:

$$
\Theta B_{1}^{\star}-A_{11}^{\star} \frac{\partial p_{0}}{\partial x_{1}}=Q
$$

The next subsection deals with two main particular cases: transverse or longitudinal roughness. 


\subsection{Particular cases}

- Transverse roughness: when the roughness does not depend on $y_{2}$, we have the homogenized equation, easily deduced from Equations (25)-(31)

$$
\frac{\partial}{\partial x_{1}}\left(\frac{1}{\overline{h^{-3}} Y} \frac{\partial p_{0}}{\partial x_{1}}\right)+\frac{\partial}{\partial x_{2}}\left({\overline{h^{3}}}^{Y} \frac{\partial p_{0}}{\partial x_{2}}\right)=\frac{\partial}{\partial x_{1}}\left(\Theta \frac{\overline{h^{-2}} Y}{\overline{h^{-3}} Y}\right),
$$

with $\Theta=\frac{1}{\bar{h}^{-2}} Y{\overline{\left(\frac{\theta_{0}}{h^{2}}\right.}}^{Y}$ and the boundary condition at the supply groove, deduced from Equation (39), should be read as:

$$
\Theta \frac{\overline{h^{-2}} Y}{{\overline{h^{-3}}}^{Y}}-\frac{1}{\bar{h}^{-3} Y} \frac{\partial p_{0}}{\partial x_{1}}=Q
$$

- Longitudinal roughness: when the roughness does not depend on $y_{1}$, we get

$$
\frac{\partial}{\partial x_{1}}\left(\bar{h}^{3} Y \frac{\partial p_{0}}{\partial x_{1}}\right)+\frac{\partial}{\partial x_{2}}\left(\frac{1}{\bar{h}^{-3} Y} \frac{\partial p_{0}}{\partial x_{2}}\right)=\frac{\partial}{\partial x_{1}}\left(\Theta \bar{h}^{Y}\right)
$$

with $\Theta=\frac{{\overline{\theta_{0} h}}^{Y}}{\bar{h}^{Y}}$, and the boundary condition at the supply groove should be read as:

$$
\Theta \bar{h}^{Y}-{\overline{h^{3}}}^{Y} \frac{\partial p_{0}}{\partial x_{1}}=Q
$$

\section{Oblique roughness}

Let us consider gaps that can be written as:

$$
h_{\varepsilon}(x)=h_{1}\left(x, \frac{X_{1}(x)}{\varepsilon}\right) h_{2}\left(x, \frac{X_{2}(x)}{\varepsilon}\right)
$$

with

$$
\left\{\begin{array}{l}
X_{1}(x)=\cos \gamma x_{1}+\sin \gamma x_{2} \\
X_{2}(x)=-\sin \gamma x_{1}+\cos \gamma x_{2}
\end{array}\right.
$$

which allows us to take into account oblique roughness (with $h_{2} \equiv 1$ for instance). The idea is to introduce a change of coordinates so that the assumption of Section B.2 on the roughness form in the new coordinates system is valid. The first step is to rewrite Equation (1) in the $X$ coordinates:

$$
\sum_{i=1}^{2} \frac{\partial}{\partial X_{i}}\left(h_{\varepsilon}^{3} \frac{\partial p}{\partial X_{i}}\right)=\left(\frac{\partial \theta h_{\varepsilon}}{\partial X_{1}} \cos \gamma-\frac{\partial \theta h_{\varepsilon}}{\partial X_{2}} \sin \gamma\right)
$$


Working now in the $X$ coordinates and using the operators defined in Section B.2 (up to the writing in the $X$ coordinates), we apply the asymptotic expansion technique to the earlier equation. With the formal asymptotic expansion used in Section B.2, we have in the $(X, y)$ coordinates (with $y=X / \varepsilon$ ):

$$
\begin{gathered}
A_{1} p_{0}=0 \\
A_{1} p_{1}+A_{2} p_{0}=B_{1}^{1} \theta_{0} \cos \gamma-B_{1}^{2} \theta_{0} \sin \gamma \\
A_{1} p_{2}+A_{2} p_{1}+A_{3} p_{0}=B_{2}^{1} \theta_{0} \cos \gamma-B_{2}^{2} \theta_{0} \sin \gamma .
\end{gathered}
$$

As in Section B.2, $p_{0}$ only depends on the $X$ variable. Equation (41) allows us to determine $p_{1}$ :

$$
p_{1}(X, y)=\chi_{1}^{0}(X, y) \cos \gamma-\chi_{2}^{0}(X, y) \sin \gamma-w_{1}(X, y) \frac{\partial p_{0}}{\partial X_{1}}(X)-w_{2}(X, y) \frac{\partial p_{0}}{\partial X_{2}}(X)
$$

Then, putting the earlier expression into Equation (42) gives:

$$
\sum_{i, j} \frac{\partial}{\partial X_{i}}\left(a_{i j}^{\star} \frac{\partial p_{0}}{\partial X_{j}}\right)=\frac{\partial}{\partial X_{1}}\left(b_{11}^{0} \cos \gamma+b_{12}^{0} \sin \gamma\right)+\frac{\partial}{\partial X_{2}}\left(b_{21}^{0} \cos \gamma+b_{22}^{0} \sin \gamma\right),
$$

where the coefficients, which are easily computed as in Section B.2, are given by $(i, j=1,2, i \neq j)$ :

$$
\begin{aligned}
& a_{i i}^{\star}={\overline{h^{3}}}^{Y}-{\overline{h^{3} \frac{\partial w_{i}}{\partial y_{i}}}}^{Y}={\frac{{\overline{h_{j}^{3}}}^{Y}}{{\overline{h_{i}^{-3}}}^{Y}},}_{{a_{i j}^{\star}}=-\bar{h}^{3} \frac{\partial w_{j}}{\partial y_{i}}}^{Y}=0,
\end{aligned}
$$

and also $(i, j=1,2, i \neq j)$

$$
\begin{aligned}
& b_{i i}^{0}={\overline{\theta_{0} h}}^{Y}-{\overline{h^{3} \frac{\partial \chi_{i}^{0}}{\partial y_{i}}}}^{Y}=\frac{1}{{\overline{h_{i}^{-3}}}^{Y}}{\overline{\left(\frac{\theta_{0} h_{j}}{h_{i}^{2}}\right)}}^{Y}, \\
& b_{i j}^{0}=-\bar{h}^{3} \frac{\partial \chi_{i}^{0}}{\partial y_{j}}
\end{aligned}
$$

Finally, as in Section B.2, defining the quantities

$$
\begin{aligned}
b_{i}^{\star} & =\frac{{\overline{h_{i}^{-2}}}^{Y}}{{\overline{h_{i}^{-3}}}^{Y}} \bar{h}_{j}^{Y}, \\
\Theta_{i} & =\frac{1}{{\overline{h_{j}}}^{Y} \overline{h_{i}^{-2}} Y} \overline{\left(\frac{\theta_{0} h_{j}}{h_{i}^{2}}\right)} Y
\end{aligned}
$$


one has $b_{i i}^{0}=\Theta_{i} b_{i}^{\star}$, with $p_{0}\left(1-\Theta_{i}\right)=0$ and $0 \leq \Theta_{i} \leq 1$.

Finally, going back to the initial $x$ coordinates, one gets the following homogenized problem:

$$
\begin{gathered}
\sum_{i, j} \frac{\partial}{\partial x_{i}}\left(A_{i j}^{\star} \frac{\partial p_{0}}{\partial x_{j}}\right)=\left(\frac{\partial B_{1}^{0}}{\partial x_{1}}+\frac{\partial B_{2}^{0}}{\partial x_{2}}\right), \\
p_{0} \geq 0 \\
0 \leq \Theta_{i} \leq 1, \quad(i=1,2), \\
p_{0}\left(1-\Theta_{i}\right)=0, \quad(i=1,2),
\end{gathered}
$$

with the left hand-side coefficients:

$$
\begin{aligned}
& A_{11}^{\star}=a_{11}^{\star}-\left(a_{11}^{\star}-a_{22}^{\star}\right) \sin ^{2} \gamma, \\
& A_{22}^{\star}=a_{22}^{\star}+\left(a_{11}^{\star}-a_{22}^{\star}\right) \sin ^{2} \gamma, \\
& A_{12}^{\star}=A_{21}^{\star}=\left(a_{11}^{\star}-a_{22}^{\star}\right) \sin \gamma \cos \gamma,
\end{aligned}
$$

and the right hand-side member:

$$
\begin{aligned}
& B_{1}^{0}=\Theta_{1} b_{1}^{\star}-\left(\Theta_{1} b_{1}^{\star}-\Theta_{2} b_{2}^{\star}\right) \sin ^{2} \gamma \\
& B_{2}^{0}=\quad\left(\Theta_{1} b_{1}^{\star}-\Theta_{2} b_{2}^{\star}\right) \sin \gamma \cos \gamma,
\end{aligned}
$$

the coefficients $a_{i i}^{\star}, b_{i}^{\star}(i=1,2)$ being given by Equation (43) ${ }^{1}$ and Equation $(47)^{1}$ in the $x$ coordinates. The link between the "microscopic saturation" $\theta_{0}$ and the two "macroscopic saturations" $\Theta_{i}(i=1,2)$ is given by Equation $(48)^{1}$.

At first glance, Equation (49) is very similar to (24). A major difference however is the anisotropic aspect of the saturation with two saturation functions $\Theta_{i}(i=1,2)$, one for each direction.

From a mathematical point of view, it is not clear wether the system of Equations (49)-(52) is a closed one or not: is a supplementary equation needed to obtain a well-posed problem or not? Nevertheless, it can be proved that $\Theta_{1}=\Theta_{2}$ is a possible choice for a solution of the system. With this assumption, it is possible to solve Equations (49)-(52) by using the kind of algorithms as the ones used to solve Equations (1)-(4). The only difference lies in the modified coefficients and the fact that the direction of the flow is no longer the $x_{1}$ axis but an oblique one.

\section{Numerical results}

As both Equations (1) and (24) have the same mathematical feature, various algorithms (see [6,7,11, 13, 15, 18,21]) used to compute solutions of Equations (1)-(4) can be adressed for the solution of Equation (24). In this paper, we propose the characteristics method adapted to steady state problems to deal with nonlinear convection term combined with finite elements. Moreover, the nonlinear Elrod-Adams model for cavitation is treated by a duality method. The combination of these numerical techniques has been explained and successfully applied by Bayada, Chambat and Vazquez in [22].

\footnotetext{
${ }^{1}$ to be translated in the $x$ coordinates
} 


\subsection{Computation of homogenized coefficients}

We consider effective gaps defined with either transverse or longitudinal roughness patterns.

Table 1 summarizes homogenized coefficients obtained for transverse and longitudinal cases:

\begin{tabular}{|c||c|c|}
\hline & Transverse roughness & Longitudinal roughness \\
\hline \hline$h(x, y)$ & $h_{s}(x)+h_{r} \sin \left(2 \pi y_{1}\right)$ & $h_{s}(x)+h_{r} \sin \left(2 \pi y_{2}\right)$ \\
\hline$A_{11}^{\star}(x)$ & $2 \frac{\left(h_{s}(x)^{2}-h_{r}^{2}\right)^{5 / 2}}{2 h_{s}(x)^{2}+h_{r}^{2}}$ & $h_{s}(x)^{3}+\frac{3}{2} h_{s}(x) h_{r}^{2}$ \\
\hline$A_{22}^{\star}(x)$ & $h_{s}(x)^{3}+\frac{3}{2} h_{s}(x) h_{r}^{2}$ & $2 \frac{\left(h_{s}(x)^{2}-h_{r}^{2}\right)^{5 / 2}}{2 h_{s}(x)^{2}+h_{r}^{2}}$ \\
\hline$B_{1}^{\star}(x)$ & $2 h_{s}(x) \frac{h_{s}(x)^{2}-h_{r}^{2}}{2 h_{s}(x)^{2}+h_{r}^{2}}$ & $h_{s}(x)$ \\
\hline
\end{tabular}

Table 1. Homogenized coefficients

The coefficients corresponding to assumption (6) can be easily obtained from the ones that are presented in Table 1, using products taking account of roughness effects in each direction.

The coefficients corresponding to oblique roughness can be obtained by using products and linear combinations of coefficients given in Table 1.

\subsection{Transverse roughness tests}

We adress the numerical simulation of journal bearing devices with axial supply of lubricant. Indeed we simulate a journal bearing device whose length is denoted $L$, the mean radius $R_{m}=\left(R_{b}+R_{j}\right) / 2, R_{b}$ and $R_{j}$ being the bearing and journal radii respectively, and the clearance is $c=R_{b}-R_{j}$. The supply flow is $Q_{R}$, the lubricant viscosity is $\mu$ and the velocity of the journal is $U$. Moreover, the roughless gap between the two surfaces is given by:

$$
H_{s}\left(X^{\prime}\right)=c\left(1+\rho \cos \left(\frac{X_{1}^{\prime}}{R_{m}}\right)\right), \quad X^{\prime}=\left(X_{1}^{\prime}, X_{2}^{\prime}\right) \in\left(0,2 \pi R_{m}\right) \times(0, L)
$$

where the eccentricity $\rho$ satisfies $0 \leq \rho<1$. The classical Reynolds problem, in real variables $X^{\prime}=\left(X_{1}^{\prime}, X_{2}^{\prime}\right)$, should be posed as follows:

$$
\begin{gathered}
\nabla \cdot\left(\frac{H_{s}^{3}}{6 \mu} \nabla P\right)=U \frac{\partial}{\partial X_{1}^{\prime}}\left(\theta H_{s}\right) \\
P \geq 0, \quad 0 \leq \theta \leq 1, \quad P(1-\theta)=0,
\end{gathered}
$$

with the boundary conditions

$$
P=0 \text {, }
$$


except on the supply groove in which

$$
U \theta H_{s}-\frac{H_{s}^{3}}{6 \mu} \frac{\partial P}{\partial X_{1}^{\prime}}=Q_{R}
$$

Now let us introduce the dimensionless coordinates and quantities that provide the effective system to be solved:

$$
\begin{array}{r}
x_{1}=\frac{X_{1}^{\prime}}{R_{m}}, \quad x_{2}=\frac{X_{2}^{\prime}}{R_{m}}, h_{s}=\frac{H_{s}}{c}, \\
p=\frac{c^{2}}{6 \mu U R_{m}} P, Q=\frac{Q_{R}}{c U}, \kappa=\frac{R_{m}}{L} .
\end{array}
$$

Then, the dimensionless Reynolds problem becomes for $x \in(0,2 \pi) \times(0, \kappa)$ :

$$
\begin{gathered}
\nabla \cdot\left(h_{s}^{3} \nabla p\right)=\frac{\partial}{\partial x_{1}}\left(\theta h_{s}\right), \\
p \geq 0, \quad 0 \leq \theta \leq 1, \quad p(1-\theta)=0,
\end{gathered}
$$

with the boundary conditions

$$
\theta h_{s}-h_{s}^{3} \frac{\partial p}{\partial x_{1}}=Q
$$

on the boundary corresponding to the dimensionless supply groove (namely $\{0\} \times(0, \kappa)$ ), and the condition

$$
p=0,
$$

on the other boundaries. The roughless gap is now $h_{s}(x)=1+\rho \cos \left(x_{1}\right)$. For the numerical tests, we have worked on the dimensionless equations, with the following data:

- $\kappa=1$, i.e. $R_{m}=L$.

- The domain being $(0,2 \pi) \times(0,1)$, the rough dimensionless gap is given by:

$$
h(x, x / \varepsilon)=h_{s}(x)+h_{r}(x / \varepsilon)=1+\rho \cos \left(x_{1}\right)+(1-\rho) \tau \sin \left(2 \pi \frac{x_{1}}{\varepsilon}\right),
$$

with $\rho=0.75, \tau=0.7, h_{s}$ (respectively $h_{r}$ ) denoting the smooth (respectively rough) contribution to the gap. - The dimensionless flow at the supply groove is $Q=\theta_{i n} h_{s}(0)$ with $\theta_{i n}=0.4571$.

For various values of $\varepsilon$, FIG. 2 represents the behavior of both pressure and saturation. In particular, it justifies the formal asymptotic expansion used in Section B.2. Three main facts have to be observed: 

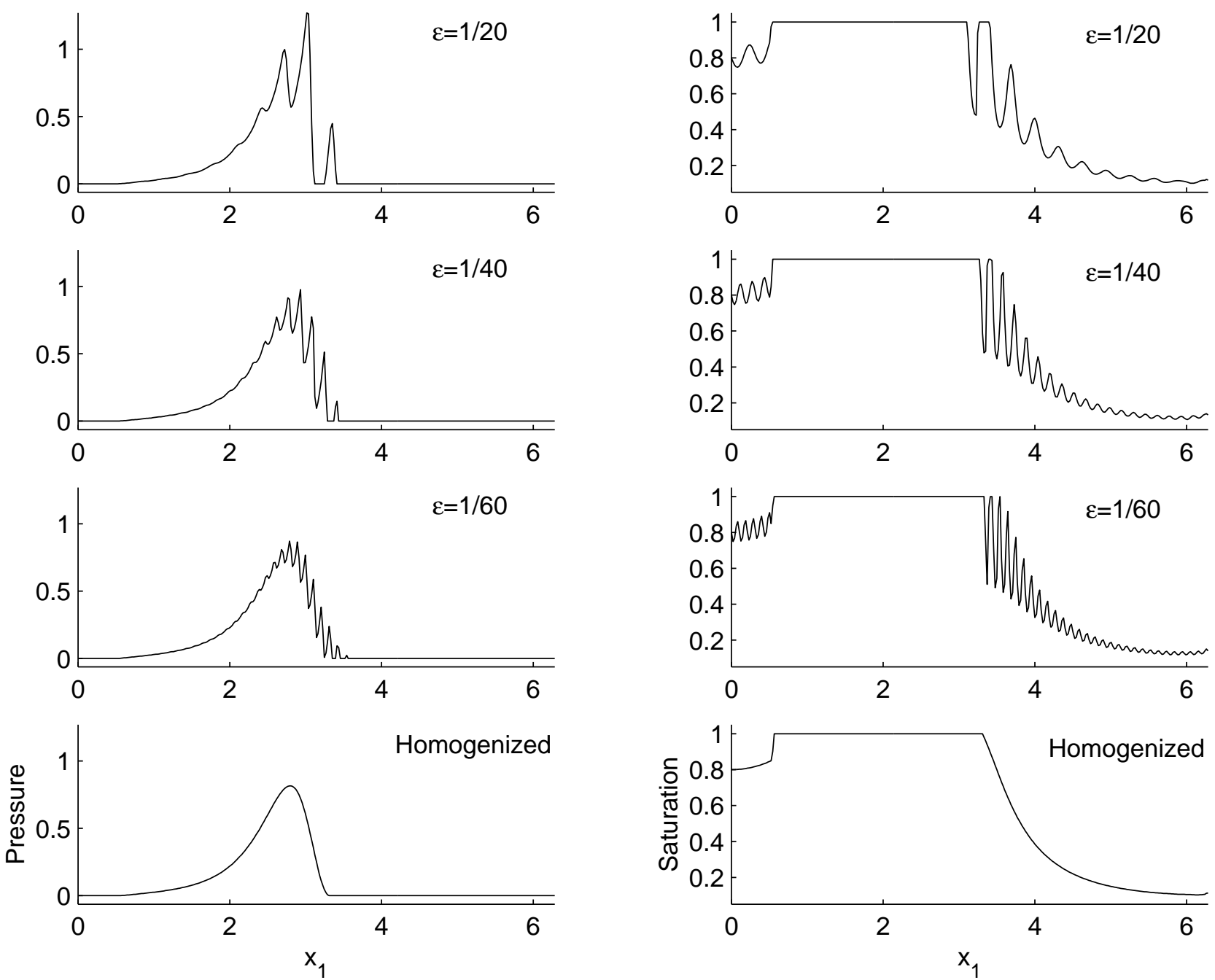

Figure 2. Pressure and saturation at $x_{2}=0.5$ for different roughness periods

$1 /$ The oscillations of the pressure tend to vanish, thus showing that $p$ tends to a smooth limit pressure (i.e. $p_{0}(x)$ ).

$2 /$ The oscillations of the saturation do not vanish; the gradient tends to explode. Thus, $\theta(x)$ behaves like a function which depends on both slow and fast variables (i.e. $\left.\theta_{0}(x, y)\right)$.

$3 /$ The existence of two cavitation areas at both extremities of the bearing (starvation phenomenon).

FIG.2 allows us not only to compare more precisely the convergence of the pressure to the homogenized one, but also to observe the behaviour of the saturation. The homogenized saturation may be viewed as an average, with respect to $y$, of the microsaturation weighted by roughness parameters. 


\subsection{Two dimensional roughness effects}

The only difference with the previous subsection lies in the definition of the dimensionless gap $h(x, y)$ defined by Assumption 6, other data being unchanged:

$$
\begin{aligned}
& h_{1}\left(x, y_{1}\right)=1+0.5 \cos \left(x_{1}\right)+0.35 \sin \left(2 \pi y_{1}\right) \\
& h_{2}\left(x, y_{2}\right)=1+0.35 \sin \left(2 \pi y_{2}\right) .
\end{aligned}
$$

FIG. 3 represents the pressure at a fixed $x_{1}$ (notice that the corresponding saturation figure is omitted, since there is nearly no cavitation).

FIG.4 and 5 represent pressure and saturation at a fixed $x_{2}$, for various values of $\varepsilon$ as well as the homogenized

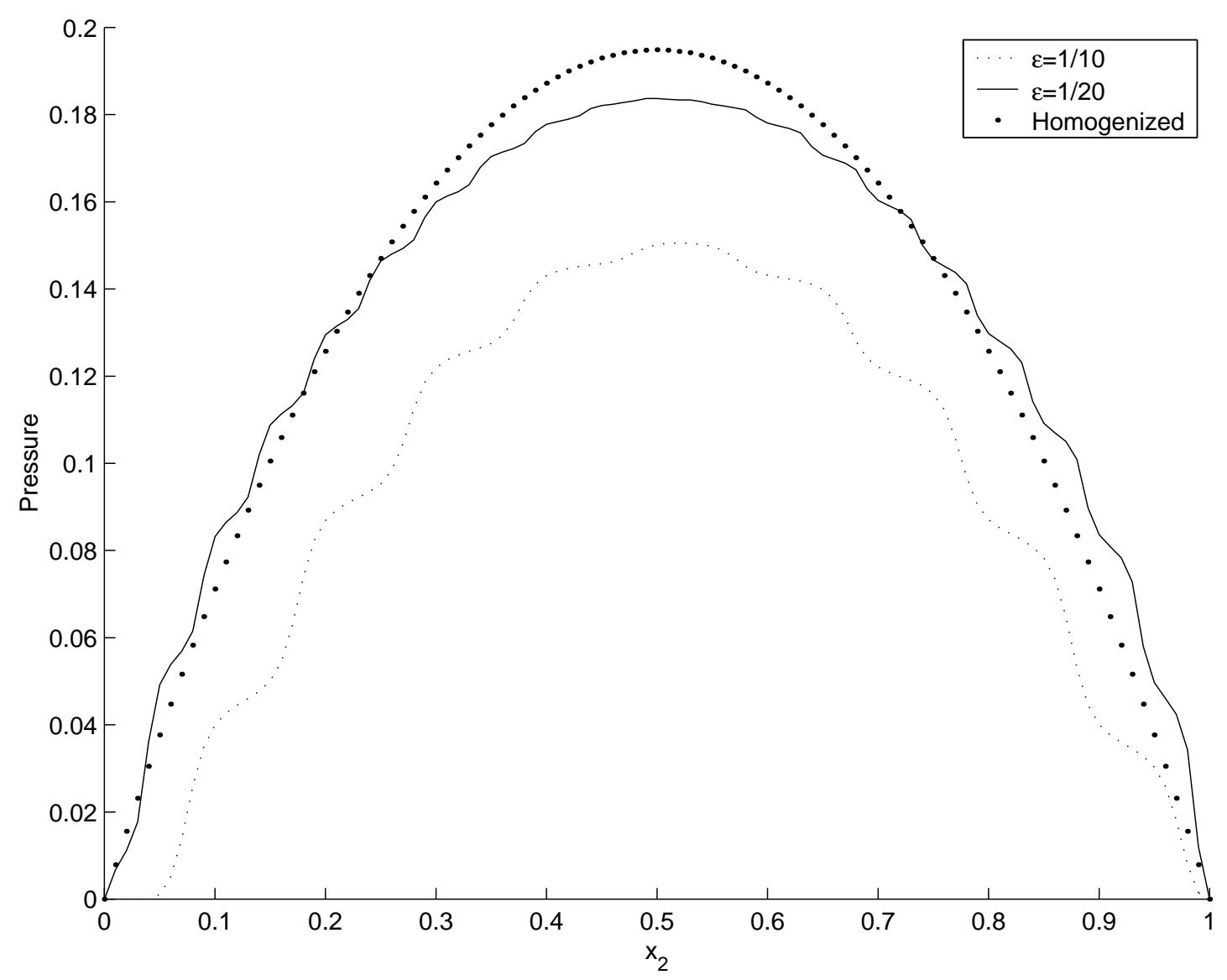

Figure 3. Hydrodynamic pressure with 2D roughness patterns at $x_{1}=2.639$

curves. Due to the number of discretized elements for solving the real problem, it is difficult to compute solutions for values of $\varepsilon$ smaller than $1 / 20$. However, the convergence for the pressure is observed in both directions, and the same comments as in the transverse roughness case can be made. 


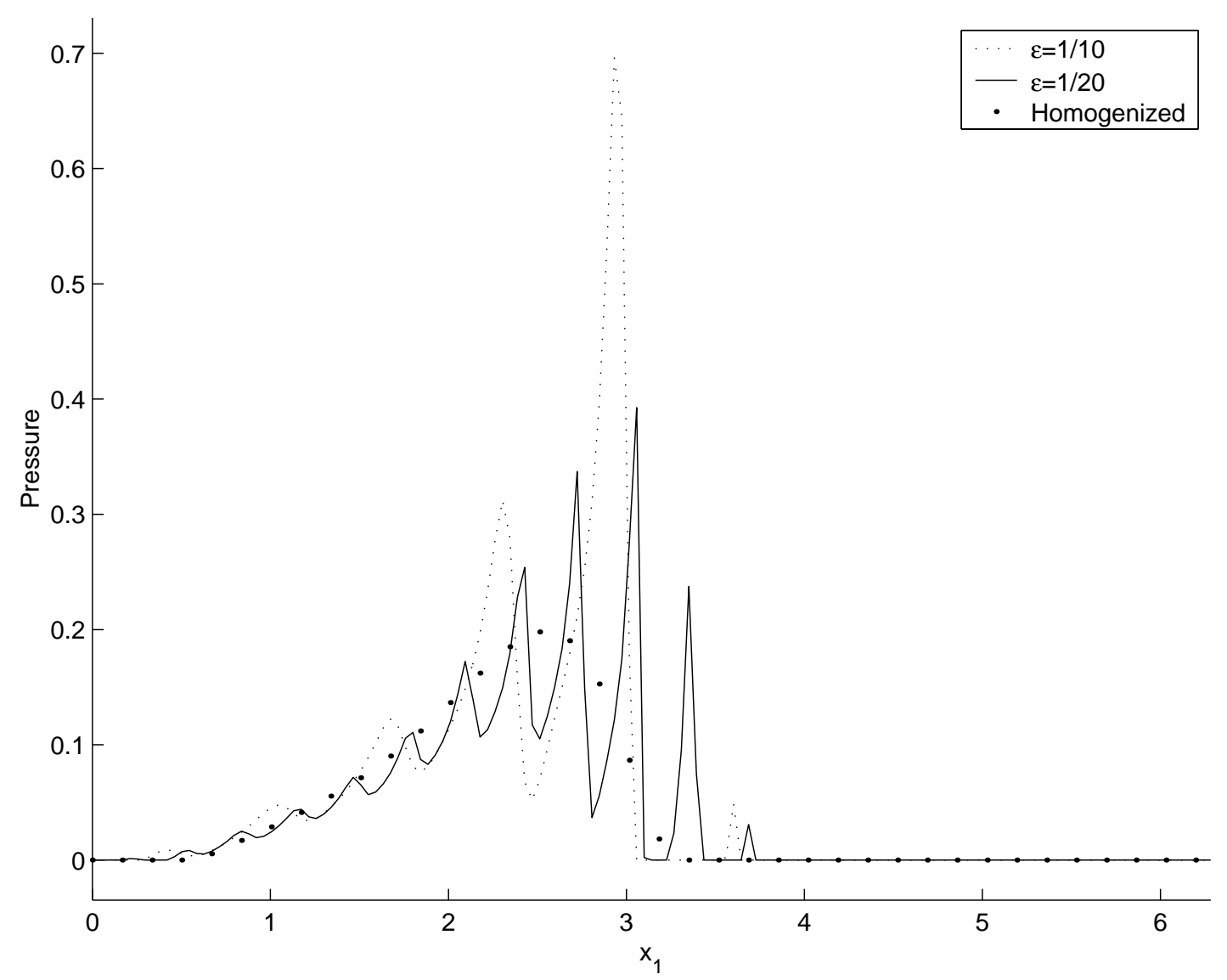

Figure 4. Hydrodynamic pressure with $2 \mathrm{D}$ roughness patterns at $x_{2}=0.5$

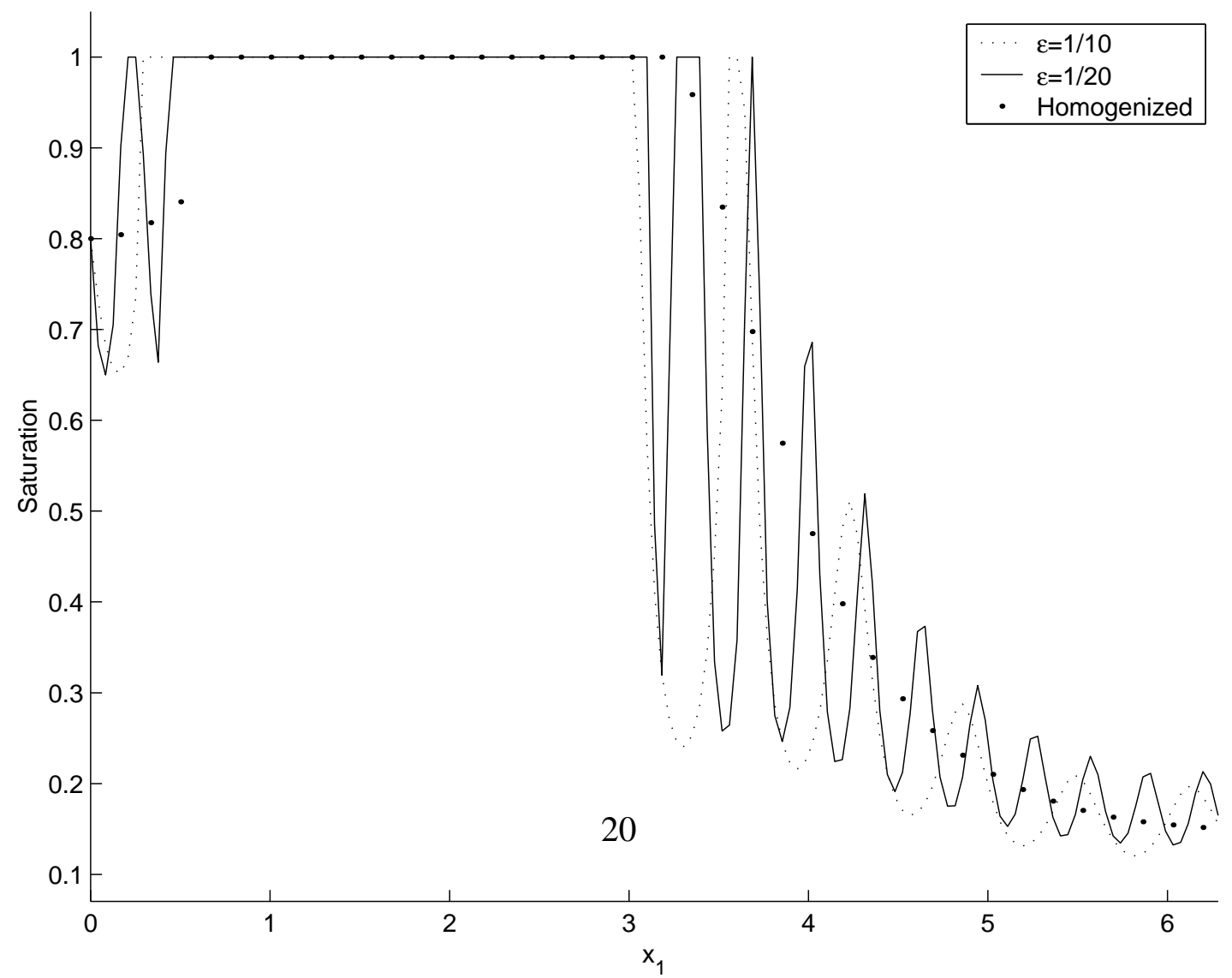

Figure 5. Hydrodynamic saturation with $2 \mathrm{D}$ roughness patterns at $x_{2}=0.5$ 


\subsection{Oblique roughness effects}

For convenience in computation, the data are not similar to the ones used in the previous subsections: considering the problem given in the real variables (see Equations (53)-(56)), we choose the following scaling process:

$$
\begin{gathered}
x_{1}=\frac{X_{1}^{\prime}}{2 \pi R_{m}}, \quad x_{2}=\frac{X_{2}^{\prime}}{2 \pi R_{m}}, \quad h_{s}=\frac{H_{s}}{c}, \\
p=\frac{c^{2}}{6 \mu U 2 \pi R_{m}} P, \quad Q=\frac{Q_{R}}{c U}, \quad \kappa=\frac{2 \pi R_{m}}{L} .
\end{gathered}
$$

Now, the dimensionless Equations (57)-(60) are considered with the following data:

- $\kappa=0.2$, i.e. $2 \pi R_{m}=0.2 L$.

- The domain being $(0,1) \times(0,0.2)$, the rough dimensionless gap is given by:

$$
h(x, x / \varepsilon)=h_{s}(x)+h_{r}(x / \varepsilon)=1+0.5 \cos \left(2 \pi \frac{e_{\gamma} \cdot x}{\varepsilon}\right),
$$

with $e_{\gamma}=(\cos \gamma, \sin \gamma), x=\left(x_{1}, x_{2}\right)$ and $\gamma=\pi / 4, h_{s}$ (respectively $h_{r}$ ) denoting the smooth (respectively rough) contribution to the gap.

- The dimensionless flow at the supply groove is $Q=\theta_{i n} h_{s}(0)$ with $\theta_{i n}=0.6$.

FIG.6 shows the behaviour of the pressure at a fixed $x_{2}$, thus clearly establishing the convergence of the pressure. FIG.7 represents the pressure on the supply line $\left(x_{1}=0\right)$, corresponding to the maximum pressure for the homogenized solution.

FIG.8 shows the evolution of the cavitated areas when $\varepsilon$ tends to 0 . Lubricated (respectively cavitated) zones are coloured in white (respectively black). For not too small values of $\varepsilon$, the direction of the cavitation streamlines is the one of the roughness pattern. This does not seem to be the case for the homogenized one.

The results point out the fact that nondiagonal terms in the left-hand side and extra term in the right-hand side of the homogenized Equation (24) or (49) are actually needed.

\subsection{Some remarks on interasperity cavitation}

In [17], Harp and Salant have proposed an average equation for modelling interasperity cavitation from JFO mass flow preserving model. Basic assumptions are the existence of a (not too small) leading value of the period of the roughness (length of correlation $\lambda$ ) and that the roughness is distributed in a somewhat stochastic way. Then the value of $\lambda$ does not disappear in the average equation obtained in [17] and allows for a description in detail of the saturation in the interasperity. However, this equation is questionable for for general roughness patterns as neither extradiagonal terms in the left hand-side nor a derivative with respect to the second direction in the right hand-side appear in the average equation, unlike to our present Equation (24). This fact has been already pointed out in [4] and is directly related to assumptions (4) in [17]. In true two dimensional roughness, it is important to take it into account, even without cavitation (see also [23]). For one dimensional roughness as the one numerically studied in [17], it is well known that these additional terms no longer exist, so that some comparison can be made between the two approaches.

FIG.9 describes numerical results linked to Harp and Salant's comments (in particular Example 2, p. 141 in [17]). The data are the following ones: 


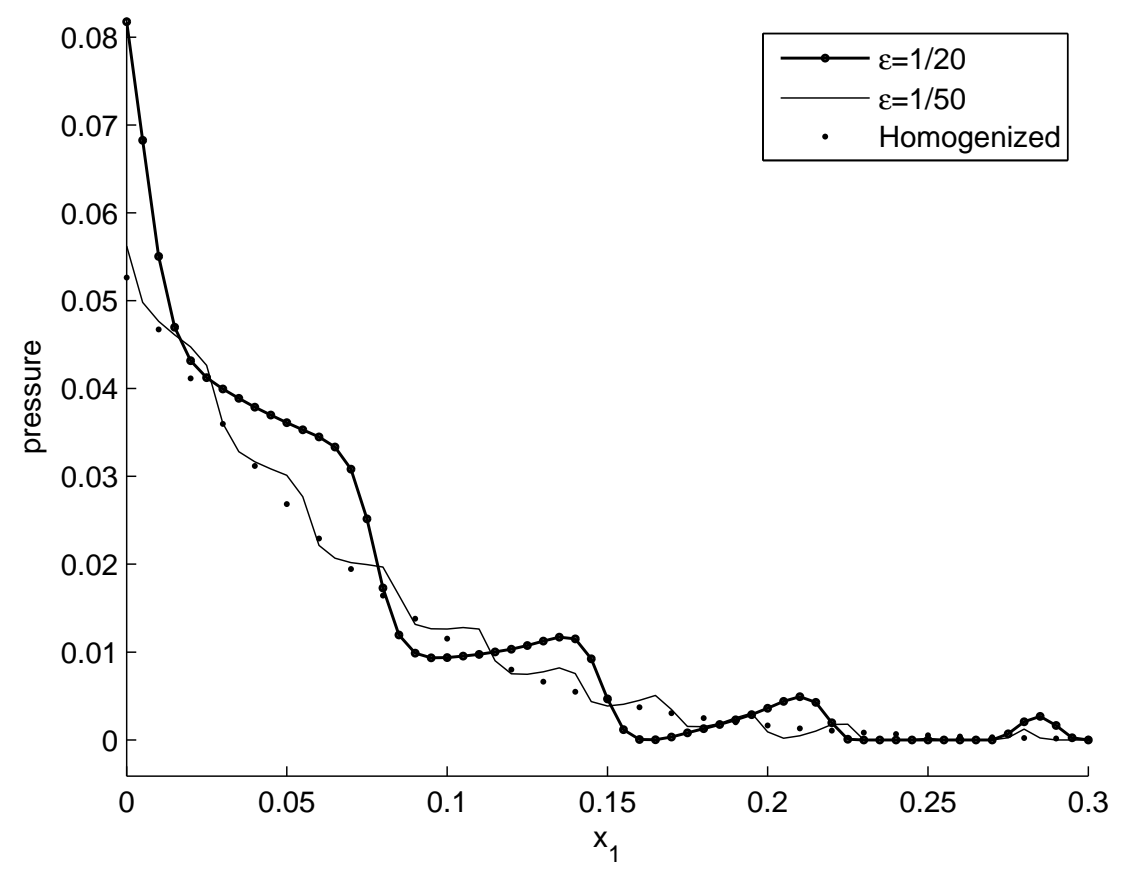

Figure 6. Hydrodynamic pressure for oblique roughness patterns at $x_{2}=0.1$

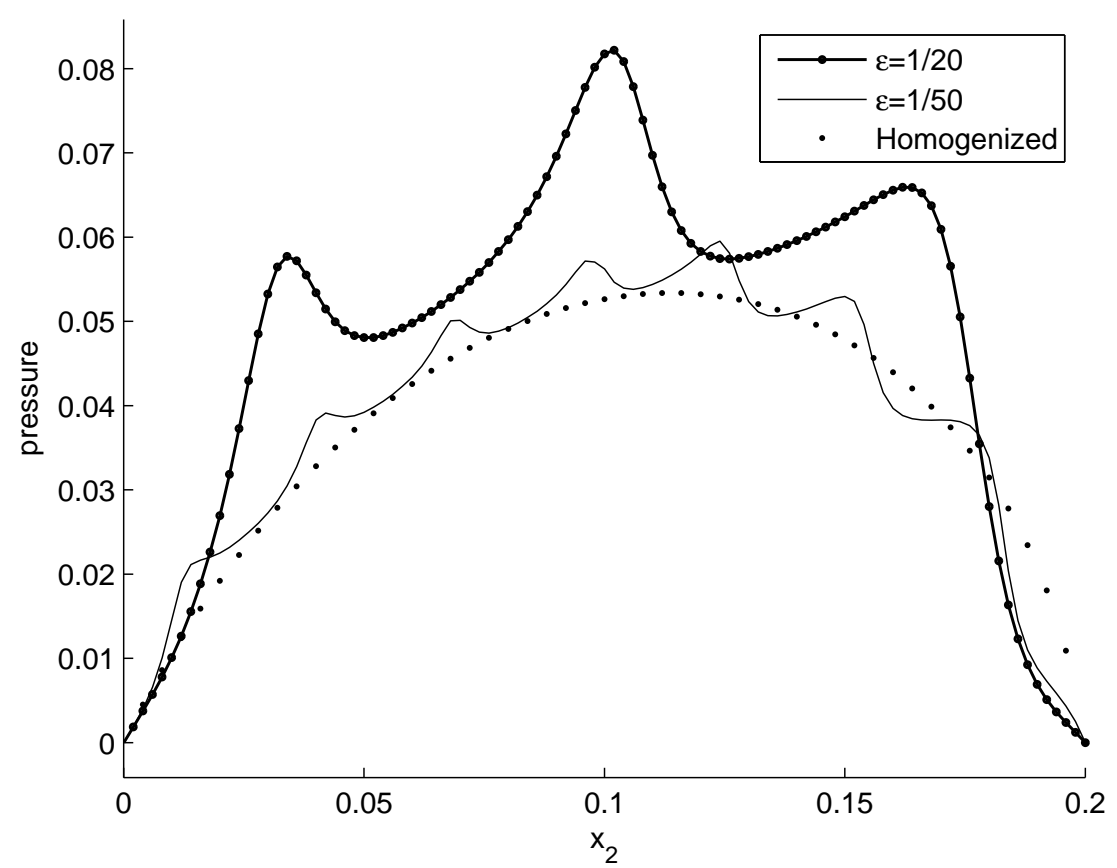

Figure 7. Hydrodynamic pressure for oblique roughness patterns at $x_{1}=0$ 

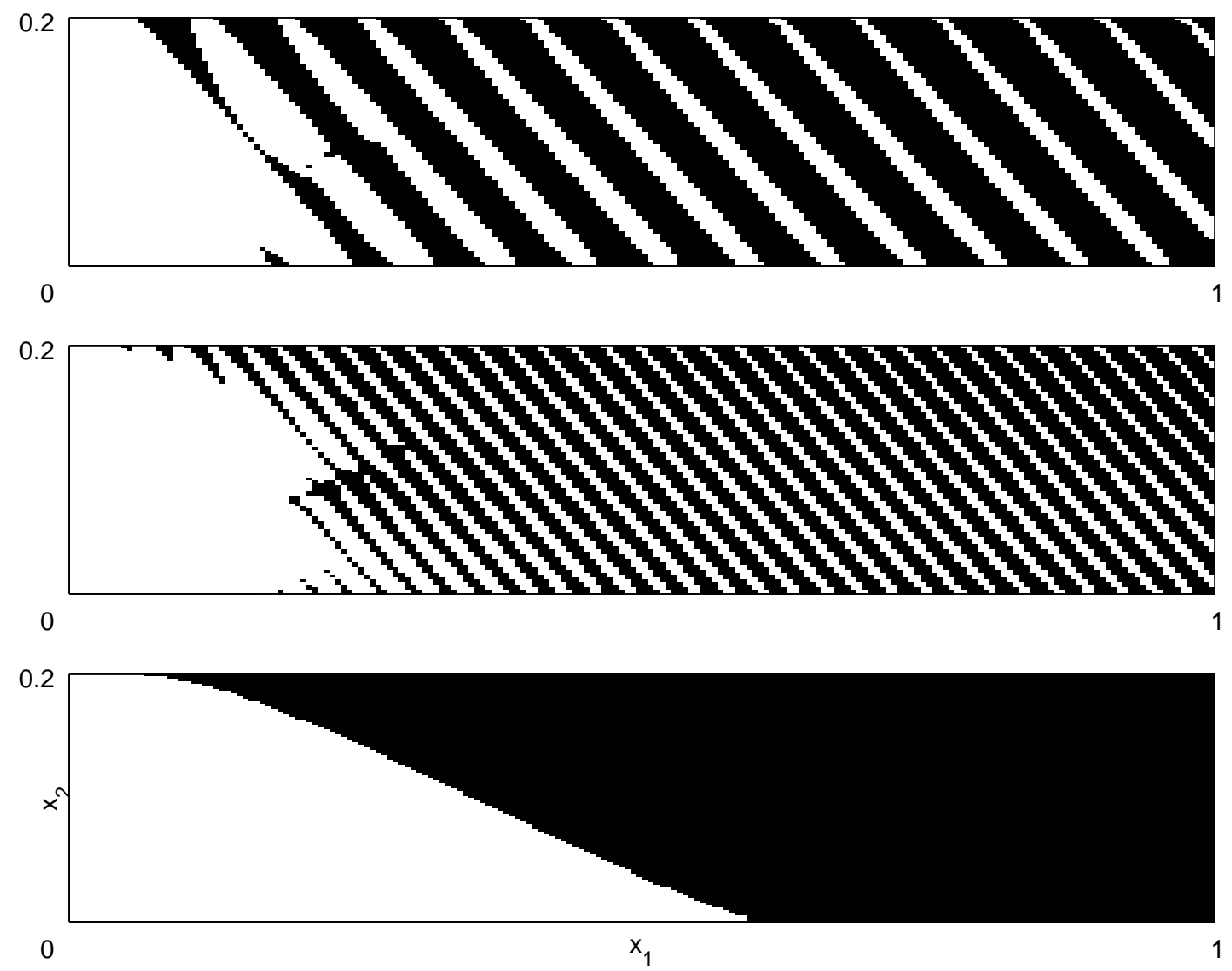

Figure 8. Lubricated [white] and cavitated [black] areas for different values of $\varepsilon: 1 / 20,1 / 50$, homogenized

- The domain is a small square bearing $] 0, l[\times] 0, l\left[\right.$ whose area is $l^{2}=0.36 \mathrm{~mm}^{2}$.

- Periodic boundary conditions are placed on $x_{1}=0$ and $x_{1}=l$.

- Pressure is imposed on other sides: $p=1.10^{5} \mathrm{~Pa}$ on $x_{2}=0$ and $p=6.10^{5} \mathrm{~Pa}$ on $x_{2}=l$.

- The effective gap is given by:

$$
h_{\varepsilon}(x)=c\left(1+0.5 \cos \left(\frac{2 \pi}{l} \frac{x_{1}}{\varepsilon}\right)\right)
$$

with $c=9 \cdot 10^{-6} \mathrm{~m}$.

- The viscosity is $\mu=0.2$ N.m.s ${ }^{-2}$.

- The velocity is $U=1 \mathrm{~m} . \mathrm{s}^{-1}$.

FIG.9 describes on the right-hand side the evolution of the saturation as a function of $\varepsilon$. The related cavitated area consists of a set of elements whose width is thiner with epsilon and whose number is proportional to $1 / \varepsilon$. In the homogenized case, the cavitation disappears.

Comparing with the results obtained in [17], we can observe that averaging the pressure in the $x_{1}$ direction gives the same kind of curves. Moreover, when $\varepsilon$ tends to 0 , the results are identical with both approaches, as the jump of the pressure at the boundary, introduced in [17], decreases with an order $\varepsilon$. 

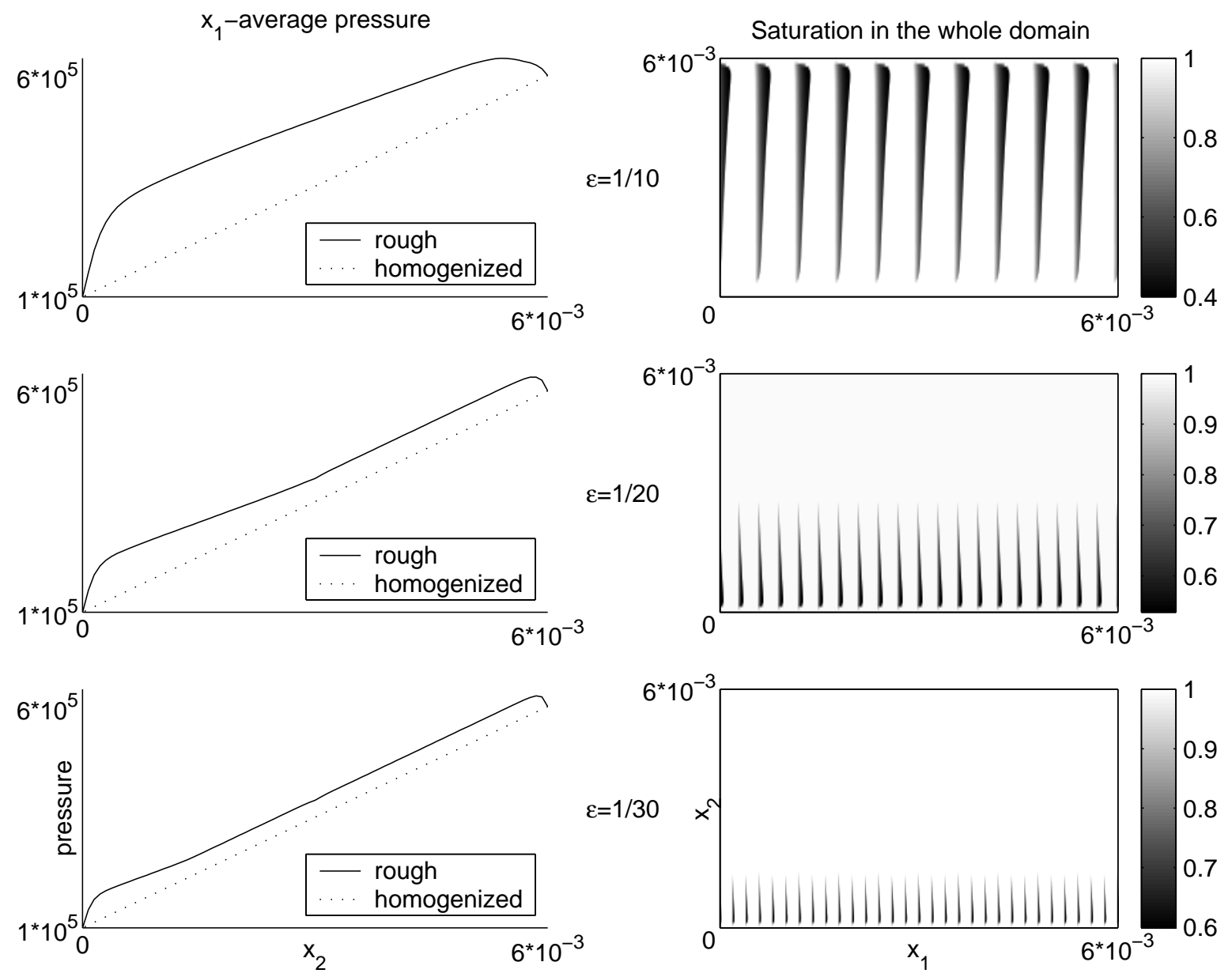

Figure 9. Average pressure and cavitated areas with interasperity

\section{Conclusion}

A solution procedure for deterministic periodic roughness computation has been developped. The procedure uses homogenization multiscale approach and rigorously takes mass flow conservation into account. Classical JFO algorithms can easily be extended to numerically compute the solution of the homogenized Reynolds equation for transverse, longitudinal, oblique and even some two dimensional roughness.

However, further mathematical developments are needed to cope with general two dimensional roughness due to anisotropic effects on the saturation.

\section{REFERENCES}

[1] Christensen, D. G., and Tonder, K., 1971. "The hydrodynamic lubrication of rough bearing surfaces of finite width". ASME J. Lub. Technol., 93 , pp. 324-330.

[2] Patir, N., and Cheng, H. S., 1978. "An average flow model for determining effects of three-dimensional roughness on partial hydrodynamic lubrication”. ASME J. Lubrication Technol., 100, pp. 12-17.

[3] Siripuram, R. B., and Stephens, L. S., 2004 (to appear). "Effect of deterministic asperity geometry on 
hydrodynamic lubrication”. ASME J. of Tribology .

[4] Bayada, G., and Faure, J.-B., 1989. "A double-scale analysis approach of the Reynolds roughness. Comments and application to the journal bearing". ASME J. of Tribology, 111 , pp. 323-330.

[5] Jai, M., and Bou-Saïd, B., 2002. "A comparison of homogenization and averaging techniques for the treatment of roughness in slip-flow-modified Reynolds equation". ASME J. of Tribology, 124, pp. 327-335.

[6] Dowson, D., Miranda, A. A. S., and Taylor, C., 1984. "Implementation of an algorithm enabling the determination of film rupture and reformation boundaries in a film bearing". In Proceedings of 10th Leeds-Lyon Symposium of Tribology, Butterworths, U.K., Paper III (ii).

[7] Bayada, G., and Chambat, M., 1986. "Sur quelques modélisations de la zone de cavitation en lubrification hydrodynamique”. J. Méc. Théor. Appl., 5 (5) , pp. 703-729.

[8] Bayada, G., and Chambat, M., 1988. "New models in the theory of the hydrodynamic lubrication of rough surfaces". ASME J. of Tribology, 110 , pp. 402-407.

[9] Floberg, L., and Jakobsson, B., 1957. "The finite journal bearing considering vaporization". Transactions of Chalmers University of Technology, Gutenberg, Sweden, 190.

[10] Olsson, K. O., 1965. "Cavitation in dynamically loaded bearing". Transactions of Chalmers University of Technology, Guthenberg, Sweden, 308 .

[11] Elrod, H. G., and Adams, M. L., 1975. "A computer program for cavitation”. Cavitation and related phenomena in lubrication - Proceedings - Mech. Eng. Publ. ltd, pp. 37-42.

[12] Elrod, H. G., 1981. "A cavitation algorithm”. ASME J. Lubrication Technol., 103 , pp. 350-354.

[13] Payvar, P., and Salant, R. F., 1992. "A computational method for cavitation in a wavy mechanical seal". ASME J. of Tribology, 114 , pp. 119-204.

[14] Brewe, D. E., 1986. "Theoretical modeling of vapor cavitation in dynamically loaded journal bearings". ASME J. Lub. Technol., 108 , pp. 628-638.

[15] Vijayaraghavan, D., and Keith, T. G., 1990. "An efficient, robust, and time accurate numerical scheme applied to a cavitation algorithm". ASME J. of Tribology, 112 , pp. 44-51.

[16] Kistler, A. L., Cheng, H. S., Nivatvongs, K., and Ozakat, I., 1980. "Cavitation phenomenon in face seals". ONR Contract N00014-79-0007.

[17] Harp, S. H., and Salant, R. F., 2001. "An average flow model of rough surface lubrication with inter-asperity cavitation". ASME J. of Tribology, 123 , pp. 134-143.

[18] Kumar, A., and Booker, J. F., 1991. "A finite element cavitation algorithm”. ASME J. of Tribology, 113 (2), pp. 276-86.

[19] Shi, F., and Salant, R. F., 2000. "A mixed soft elastohydrodynamic lubrication model with interasperity cavitation and surface shear deformation". ASME J. of Tribology, 122 , pp. 308-316.

[20] Bayada, G., Martin, S., and Vázquez, C., 2005. "Effets d'anisotropie par homogénéisation dans un problème à frontière libre". C. R. Math. Acad. Sci. Paris, 340 (7) , pp. 541-546.

[21] Hooke, C. J., 1998. "The behaviour of low-amplitude surface roughness under line contacts". Proc. Instn. Mech. Engrs., 213 , pp. 275-285.

[22] Bayada, G., Chambat, M., and Vázquez, C., 1998. "Characteristics method for the formulation and computation of a free boundary cavitation problem". J. Comput. Appl. Math., 98 (2), pp. 191-212.

[23] Buscaglia, G., and Jai, M., 2004. "Homogenization of the generalized Reynolds equation for ultra-thin gas films and its resolution by FEM". ASME J. of Tribology, 126 , pp. 547-552. 